\title{
Standardization of Sonographic Diaphragm Thickness Evaluations in Healthy Volunteers
}

\author{
Raúl Carrillo-Esper MD, Ángel Augusto Pérez-Calatayud MD, Emilio Arch-Tirado MD PhD, \\ Manuel Alejandro Díaz-Carrillo MD, Eduardo Garrido-Aguirre MD, Rafael Tapia-Velazco MD, \\ Carlos Alberto Peña-Pérez MD, Isis Espinoza-de los Monteros MD, \\ José Martín Meza-Márquez MD, Oscar Iván Flores-Rivera MD, \\ Adriana Denise Zepeda-Mendoza MD, and Teresa de la Torre-León MD
}

\begin{abstract}
BACKGROUND: B-mode ultrasound can be used to measure diaphragm thickness at the zone of apposition. We believe it is necessary to develop normal values for diaphragm thickness at rest in a large group of healthy subjects and compare them with international results. METHODS: Ultrasound measurements of diaphragm thickness at expiratory rest were taken in 109 healthy individuals, with results stratified by sex, body mass index, and thorax circumference. The following methods were used for analysis and interpretation. Multivariable databases with descriptive statistical analyses were made. The Pearson chi-square test was used to evaluate the distribution between variables. Additionally, mean and SD values were calculated. For standardization, the data were separated by sex within a $95 \% \mathrm{CI}$, and we calculated a $\mathrm{Z}$ test. A 95\% CI was also constructed for proportion analyses. RESULTS: One hundred nine healthy volunteers were included in the study, and the correlation between the body mass index and thorax circumference values with a Pearson chi-square test resulted in an $r=0.69$. Additionally, the average value of diaphragmatic thickness was $0.19 \pm 0.04 \mathrm{~cm}(95 \% \mathrm{CI} 0.17-0.20 \mathrm{~cm})$ for men and $0.14 \pm 0.03 \mathrm{~cm}(95 \% \mathrm{CI} 0.13-0.15 \mathrm{~cm})$ for women $(P=.001)$. There was no relation between body mass index thorax circumference, and diaphragmatic thickness. CONCLUSION: Real-time ultrasound of the diaphragm is a simple, inexpensive, and portable imaging technique that can provide qualitative anatomical information. The findings in this study show that sonographic diaphragm evaluations can be applied to the general population. Key words: diaphragm thickness, ultrasound [Respir Care 2016;61(7):920-924. (C) 2016 Daedalus Enterprises]
\end{abstract}

\section{Introduction}

The diaphragm is the major respiratory muscle that is used for quiet breathing. Diaphragmatic dysfunction can

Dr Carrillo-Esper is affiliated with the Department of Critical Care, Fundación Clinica Médica Sur, Mexico City 14050, Mexico. Drs PérezCalatayud, Díaz-Carrillo, Garrido-Aguirre, Tapia-Velazco, Peña-Pérez, Espinoza-de los Monteros, Martín Meza-Márquez, Flores-Rivera, ZepedaMendoza, and de la Torre-León are affiliated with the Department of Critical Care Medicine, Fundación Clinica Médica Sur, Mexico City 14050, Mexico. Dr Arch-Tirado is affiliated with the Research in Medical Sciences Neuro-Rehabilitation Laboratory, National Institute of Rehabilitation, Mexico City, Mexico.

The authors have disclosed no conflicts of interest.

Correspondence: Ángel Augusto Pérez-Calatayud MD, Fundación Clínica be caused by conditions that directly involve the diaphragm, such as trauma, cardiothoracic surgery, adjacent thoracic or abdominal pathology (eg, basal pulmonary atelectasis, pneumonia, or tumors), upper abdominal masses, extensive pleural or abdominal fluid, and muscular dystrophies. ${ }^{1}$

To evaluate diaphragm dysfunction, tests such as chest radiographs, video fluoroscopy, and pulmonary function testing are inconclusive or suggest neuromuscular respiratory impairment. Additionally, phrenic nerve conduction studies and needle electromyogram of the

Médica Sur, Puente de Piedra 150, Col. Toriello Guerra Delegación
Tlalpan, Mexico City 14050, Mexico. E-mail: gmemiinv@gmail.com.

DOI: $10.4187 /$ respcare.03999 


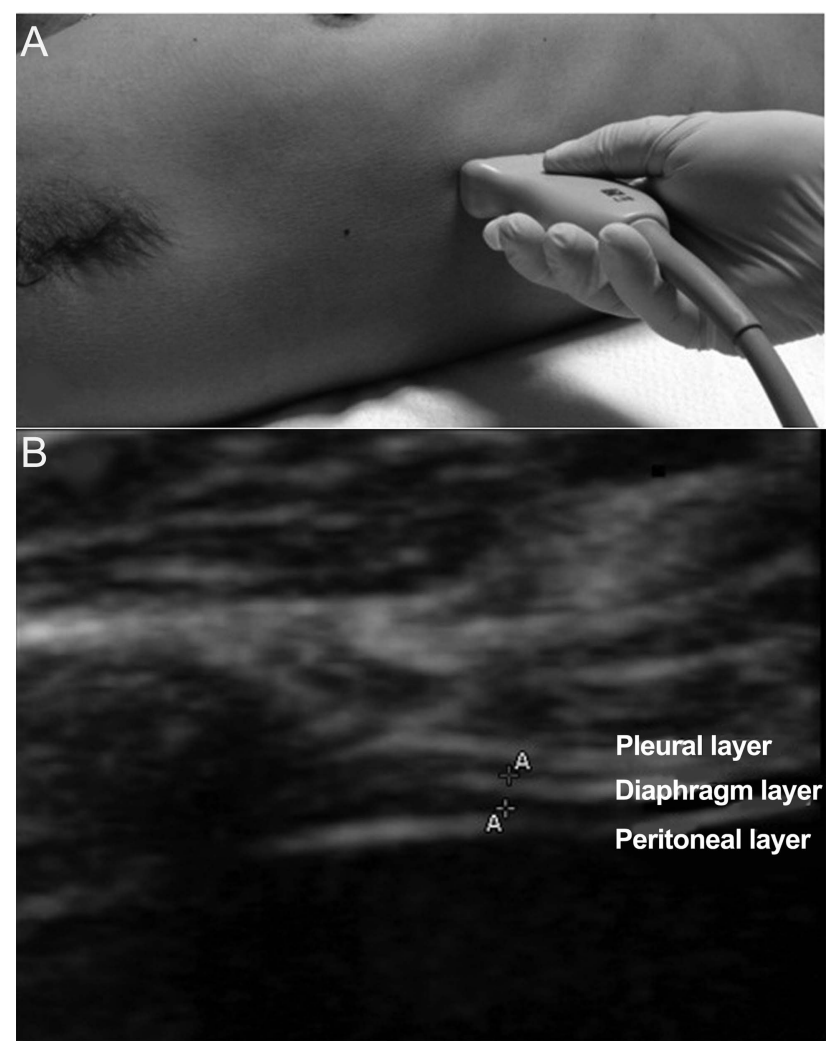

Fig. 1. A: The probe position for a B-mode diaphragmatic thickness measurement in the zone of apposition with a $10-15-\mathrm{MHz}$ probe. B: A diaphragm thickness image displaying the following different layers from top to bottom: echogenic diaphragmatic layer (pleural), non-echogenic central layer (diaphragm), and echogenic layer (peritoneal).

diaphragm are often helpful in this setting but may be technically difficult or relatively contraindicated..$^{2-4}$

Sonographic diaphragm evaluations have recently started to gain popularity in the ICU as specific needs for assessing diaphragmatic function have arisen in many clinical situations. Ultrasound has also been used to evaluate diaphragmatic thickness in the zone of apposition from the diaphragm to the rib cage. The zone of apposition is the chest wall area where the abdominal contents reach the lower rib cage (Fig. 1A). In this area, the diaphragm is observed as a structure made of 3 distinct layers (Fig. 1B). These include a non-echogenic central layer that is bordered by 2 echogenic layers, the peritoneum and the diaphragmatic pleurae. ${ }^{5}$ Weaning failure, a prevalent clinical problem in critically ill adult patients, is the failure to wean from mechanical ventilation. Mechanical ventilation is associated with decreased muscle weight and alterations in contractile properties of the diaphragm within $48 \mathrm{~h}$ of intubation. ${ }^{6,7}$ This has led to suspicions that diaphragm dysfunction may contribute to weaning failure, even in patients with no obvious reason to suspect phrenic nerve or diaphragm pathology. The Levine study ${ }^{8}$ indicated that

\section{QUICK LOOK}

\section{Current knowledge}

Ultrasound has been used to evaluate diaphragmatic thickness. Research on diaphragm ultrasound is constantly evolving, with the latest study indicating its possible applications previously not accessible for diaphragm evaluation.

\section{What this paper contributes to our knowledge}

The ultrasound evaluation of diaphragm thickness proposed was standardized by sex in healthy volunteers, where differences were found in the measurements obtained from both groups. Diaphragm thickness is constant in both sexes regardless of age or body mass index, which must be taken into account for clinical decisions.

the combination of $18-69 \mathrm{~h}$ of diaphragm inactivity and mechanical ventilation was associated with marked atrophy of both slow-twitch and fast-twitch fibers in the human diaphragm. This observation strongly suggests the presence of increased proteolysis.

It has been proposed that B-mode ultrasound can be used to measure diaphragm thickness at the zone of apposition during inspiration or expiration using the intercostal approach. Thickness measured by ultrasound has been shown to correlate with direct diaphragm thickness measurements on a cadaver. ${ }^{9}$

It was necessary to standardize values for diaphragm thickness at rest in a large group of healthy subjects and to establish whether there is a relationship between demographic data such as sex, body mass index (BMI), and thorax circumference, regarding the use of validated basal measurements in ICUs.

\section{Methods}

We conducted a descriptive cross-sectional study; a sample was calculated for known variance $P=.01 .{ }^{10} \mathrm{We}$ received authorization from the local ethics committee (institutional review board: Comité de Ética e Investigación para Studios en Humanos). Healthy subjects of both sexes (55 males and 54 females) were recruited from 2 tertiary hospitals from February to August 2014, all of whom gave verbal consent and signed authorization. The exclusion criteria consisted of any history of dyspnea or generalized neuromuscular disease, such as peripheral neuropathy, myopathy, motor neuron disease, or central nervous system disease. The demographic and anthropometric data for these subjects are shown in Table 1.

Demographic data were collected, followed by ultrasound imaging of the diaphragm. Subjects were examined 
Table 1. Anthropometric Variables Between Sexes

\begin{tabular}{lcc}
\hline \hline \multicolumn{1}{c}{ Variable } & Mean $\pm \mathrm{SD}$ & $95 \% \mathrm{CI}$ \\
\hline Age, y & $25.8 \pm 6.5$ & $24.5-27.0$ \\
Age (men), y & $24.9 \pm 4.9$ & $23.6-26.2$ \\
Age (women), y & $26.3 \pm 7.5$ & $24.3-28.2$ \\
Height, cm & $167 \pm 0.97$ & $165-168$ \\
Height (men), cm & $173 \pm 0.74$ & $171-174$ \\
Height (women), cm & $159 \pm 0.57$ & $157-160$ \\
Weight, kg & $67.6 \pm 14.18$ & $65.0-70.3$ \\
Weight (men), kg & $76.8 \pm 13.24$ & $73.3-80.3$ \\
Weight (women), kg & $58.3 \pm 7.56$ & $56.2-60.3$ \\
BMI, kg/m ${ }^{2}$ & $24.1 \pm 3.62$ & $23.5-24.8$ \\
BMI (men), kg/m ${ }^{2}$ & $25.4 \pm 3.69$ & $24.4-26.4$ \\
BMI (women), kg/m ${ }^{2}$ & $22.9 \pm 3.18$ & $22.1-23.8$ \\
Thorax circumference, cm & $88.6 \pm 10.27$ & $86.6-90.5$ \\
Thorax circumference (men), cm & $94.6 \pm 8.27$ & $92.4-96.8$ \\
Thorax circumference (women), cm & $82.3 \pm 8.34$ & $80.2-84.6$ \\
\end{tabular}

$\overline{\mathrm{BMI}=\text { body mass index }}$

in a supine position. A high-resolution ultrasound machine (M-turbo, SonoSite, Mexico City, Mexico) was used, which included a $10-5-\mathrm{MHz}$ linear array transducer. Real-time ultrasound was used to identify the intercostal space. Typically, the area between the 8th and 9th intercostal space was chosen, which is just before the previous axillary line, where the diaphragm was most easily visualized (with the transducer spanning 2 ribs). The diaphragm was identified by its characteristic 3-layered appearance and location beneath the intercostal muscles and subcutaneous tissue, where the 2 facial lines outlining the diaphragm are parallel. To observe the zone of apposition of the muscle, diaphragm layer is found $0.5-2 \mathrm{~cm}$ below the costophrenic sinus, consisting of a relatively non-echogenic muscular layer bound by the echogenic membranes of the diaphragmatic pleura and peritoneum.

Three images for each position were collected and averaged to determine a thickness at resting end expiration. On each frozen B-mode image, the diaphragm thickness was measured from the center of the pleural line to the center of the peritoneal line. The images were measured on the ultrasound system itself, using the measurement tool.

\section{Statistical Analyses}

Statistical analyses were performed using SPSS (SPSS, Chicago, Illinois). For this purpose, a multivariable database was constructed. Descriptive statistical analyses were made to establish data behavior. The Pearson chi-square test was used to evaluate the distribution between variables. Mean \pm SD values for diaphragmatic thicknesses were calculated. For possible standardization, data were separated by
Table 2. Expiratory Diaphragm Thickness Confidence Intervals

\begin{tabular}{lll}
\hline \hline \multicolumn{1}{c}{ Variable } & Mean \pm SD & $95 \%$ CI \\
\hline Diaphragm thickness, cm & $0.16 \pm 0.04$ & $0.15-0.17$ \\
Diaphragm thickness (women), cm & $0.14 \pm 0.03$ & $0.13-0.15$ \\
Diaphragm thickness (men), cm & $0.19 \pm 0.04$ & $0.17-0.20$ \\
\hline
\end{tabular}

sex and constructed within a $95 \%$ CI. A Z test was calculated for diaphragmatic thicknesses. Finally, a 95\% CI was constructed for proportion analyses to establish the relationship between diaphragmatic thickness variables.

\section{Results}

One hundred nine healthy volunteers were included in the study. The average age was $25.8 \pm 6.5 \mathrm{y}$. Regarding sex, there were 55 males $(50.5 \%)$ with an average age of $24.9 \pm 4.9 \mathrm{y}$ and 54 females $(49.5 \%)$ with an average age of $26.3 \pm 7.5 \mathrm{y}$. CI were constructed with $95 \%$ reliability for age, height, weight, BMI, and thoracic circumference in the overall sample as well as the samples by sex in order to propose standards for these variables; thus, we propose to associate the intervals for diaphragmatic thickness during expiration (see Table 1).

The data for BMI and thorax circumference $(r=0.69)$ show a considerable correlation between these 2 variables and a linear regression adjustment between the BMI and thorax circumference. A positive $r$ value was noted along with a linear association between these 2 variables. The resulting slope was $<90^{\circ}$; therefore, the correspondence between these 2 variables was increased both on the ordinate and abscissa. The correlation evaluation between BMI, thorax circumference, and diaphragmatic thickness resulted in an $\mathrm{r}=0.02$; therefore, there was no correlation between these 2 variables ( $\mathrm{Ta}-$ ble 2). Mean diaphragmatic thickness \pm SD was calculated. For possible standardization, the subjects were divided by sex. The average diaphragmatic thickness value was $0.19 \pm 0.04 \mathrm{~cm}(95 \%$ CI $0.17-0.19 \mathrm{~cm})$ for men and $0.14 \pm 0.03 \mathrm{~cm}(95 \%$ CI $0.13-0.15 \mathrm{~cm})$ for women (Fig. 2 and Table 2).

To test the consistency between sex and diaphragmatic thickness, box plots were performed and evaluated for the position of the median relative to their quartiles. We found a median value of $0.19 \mathrm{~cm}$ for men and $0.14 \mathrm{~cm}$ for women and found better and more consistent data distribution in the women compared with men, in which 5 results protruded from the upper limit of the estimated quartiles. Still, the trend of the data was differentiated from the values that were obtained from the diaphragmatic thickness measurements (Fig. 3).

A proportion analysis was conducted to evaluate the relationship between BMI and diaphragmatic thickness (diaphragmatic thickness/BMI) and found no relation between 


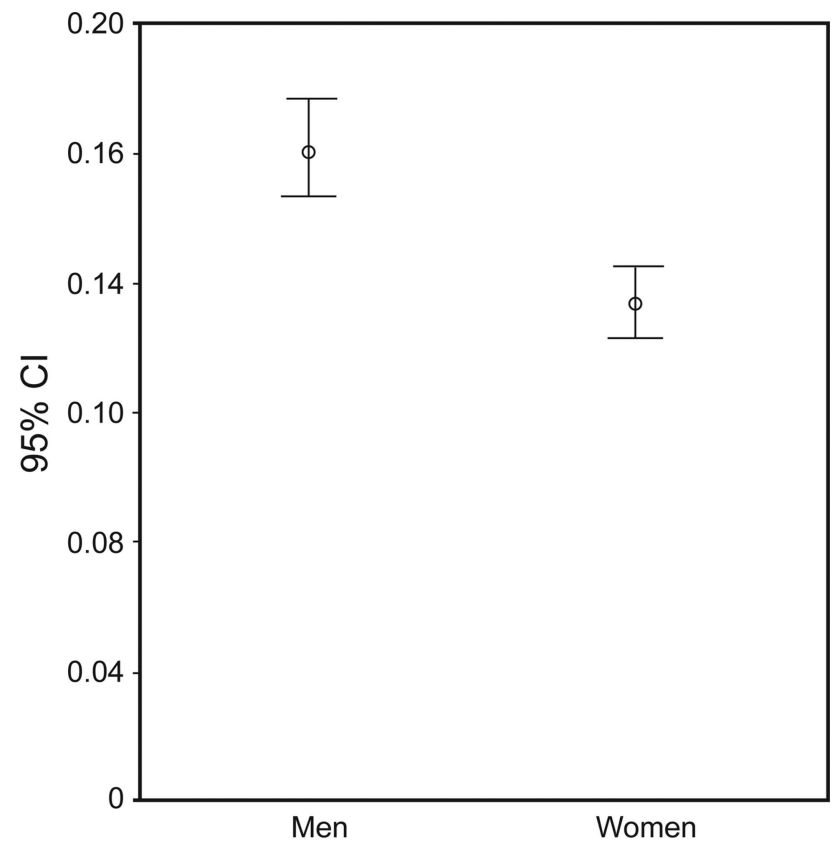

Fig. 2. The mean \pm SD values for the diaphragmatic thicknesses were calculated for possible standardization and were separated by men and women.

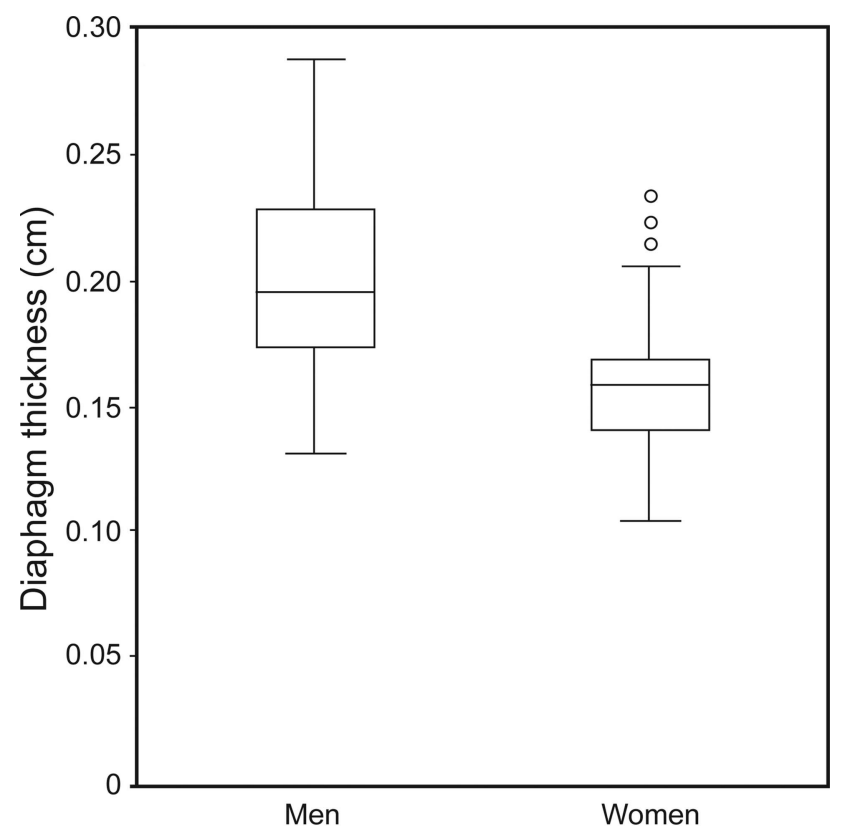

Fig. 3. The position of the median was evaluated relative to its quartiles. There was better and more consistent data distribution in men compared with women, in which 5 results protruded from the upper limit of the estimated quartiles. The average diaphragmatic thickness value was $0.19 \pm 0.04 \mathrm{~cm}(95 \% \mathrm{Cl} 0.17-0.19 \mathrm{~cm})$ for men and $0.14 \pm 0.03 \mathrm{~cm}(95 \% \mathrm{Cl} 0.13-0.15 \mathrm{~cm})(P=.001)$ for women. The upper edge of each box represents the 1st quartile, whereas the bottom edge shows the 3 rd quartile. The center lines denote the median.
Table 3. Confidence Intervals for Proportions

\begin{tabular}{lr}
\hline \hline \multicolumn{1}{c}{ Proportion } & $95 \%$ CI \\
\hline Diaphragmatic thickness/thorax circumference & {$[0-0.009]$} \\
Diaphragmatic thickness/BMI & {$[0.0086-0.02]$} \\
& \\
\hline BMI = body mass index & \\
\hline
\end{tabular}

these 2 variables. Additionally, there was also no correlation between variables for thorax circumference and diaphragmatic thickness (diaphragmatic thickness/thorax circumference). Therefore, there is no significant difference between sexes for the proportions described above; we propose one interval for both sexes (see Table 3 ).

It was established that there is a significant difference in the diaphragmatic thickness between sexes. There was a difference of $P<.001$; therefore, the intervals should be established independently. In this manner, the $95 \%$ CI for reliability in women is $0.12-0.14 \mathrm{~cm}$, and for men, it is $0.17-0.19 \mathrm{~cm}$ (see Table 2).

\section{Discussion}

Real-time ultrasound of the diaphragm is a simple, inexpensive, and portable imaging technique. The interval proposed in our study was standardized by sex. This variable is important for diaphragm measurement cut-off values. Whereas the expected relationship between BMI and thorax circumference was observed, there were no relationships between weight, BMI, or thorax circumference and the diaphragmatic thickness results. When we compared mean and median, the result was almost the same. Men had a mean of $0.19 \mathrm{~cm}$, and the median was the same, and with women, mean and median was $0.14 \mathrm{~cm}$, which indicates that the homogeneity of diaphragm thickness measured is manifested, demonstrating the reliability of the proposed intervals.

Previous reports also found no relationship between age and diaphragmatic thickness. Boon et $\mathrm{al}^{10}$ found that the lower limit of normal diaphragm thickness at end expiration or functional residual capacity is $0.15 \mathrm{~cm}$. They reported that thickness at end expiration of $>0.33 \mathrm{~cm}$ is abnormal and also found that diaphragm thickness is minimally affected by age, sex, body habitus, or smoking history. In the vast majority of normal subjects, the diaphragm is at least $0.15 \mathrm{~cm}$ thick in the zone of apposition at resting end expiration and shows a $<0.33-\mathrm{cm}$ side-toside difference in resting thickness. This is consistent with our results because in our sample, the range of diaphragm thickness was $0.12-0.19$, so it appears that the age range has no effect on the diaphragm thickness proposed in this study. 


\section{Standardization of Sonographic Diaphragm Thickness}

A few studies have addressed the subject of ultrasounds for diaphragmatic displacement and thickness measurement accuracy and reproducibility in healthy volunteers and subjects in the ICU. When measuring diaphragmatic excursions in healthy volunteers, Boussuges et $\mathrm{al}^{11}$ reported that the intra-observer reproducibility was 96 and $94 \%$, and the inter-observer reproducibility was 95 and 91\% during quiet breathing for the right and left diaphragm, respectively. Reliability data from this study were very good when care was taken to ensure that measurements were taken at the same anatomical position. However, during data collection, we observed that measurements of diaphragm thickness could be highly variable, depending on which intercostal space was chosen. Regarding this issue, Goligher et al ${ }^{12}$ made diaphragm measurements on critically ill subjects. Right hemidiaphragm thickness was obtained in $95 \%$ of attempts, demonstrating that the measurement of diaphragm thickness is highly reproducible. According to these results, we can use diaphragm thicknesses to evaluate the presence of diaphragm atrophy that is associated with mechanical ventilation, ${ }^{13-16}$ although for diaphragm function, we prefer to use functional ultrasound tests, such as diaphragmatic excursions and inspiratory and expiratory diaphragm thickness changes. ${ }^{13,16,17}$

A limitation of this study is that we did not conduct a pulmonary function test to ensure normal lung function among the study subjects. However, previous reports in smaller groups of subjects have shown that it is the thickening ratio that correlates with vital capacity and maximum inspiratory pressure. Another limitation is that this study included only healthy subjects. The value of this imaging technique in patients with preexisting conditions, including central nervous system disorders and COPD, is unknown.

\section{Conclusions}

Research on diaphragm ultrasound is constantly evolving, with the latest study indicating its possible applications for ultrasound diaphragmatic evaluation, which previously was difficult to access. ${ }^{17}$ The findings of this study show that sonographic diaphragm evaluations can be applied to the general population. Additionally, our findings are similar to those previously reported in other international studies. However, more studies are needed to evaluate the application of this technique for anatomical changes of the diaphragm in critical care and rehabilitation settings.

\section{REFERENCES}

1. Gerscovich EO, Cronan M, McGahan JP, Jain K, Jones CD, McDonald C. Ultrasonographic evaluation of diaphragmatic motion. J Ultrasound Med 2001;20(6):597-604.

2. Bolton CF, Grand'Maison F, Parkes A, Shkrum M. Needle electromyography of the diaphragm. Muscle Nerve 1992;15(6):678-681.

3. Chen R, Collins S, Remtulla H, Parkes A, Bolton CF. Phrenic nerve conduction study in normal subjects. Muscle Nerve 1995;18(3):330335 .

4. Resman-Gaspersc A, Podnar S. Phrenic nerve conduction studies: technical aspects and normative data. Muscle Nerve 2008;37(1):36-41.

5. Ayoub J, Cohendy R, Dauzat M, Targhetta R, De la Coussaye JE, Bourgeois JM, et al. Non-invasive quantification of diaphragm kinetics using m-mode sonography. Can J Anaesth 1997;44(7):739-744.

6. Le Bourdelles G, Viires N, Boczkowski J, Seta N, Pavlovic D, Aubier M. Effects of mechanical ventilation on diaphragmatic contractile properties in rats. Am J Respir Crit Care Med 1994;149(6): 1539-1544

7. Capdevila X, Lopez S, Bernard N, Rabischong E, Ramonatxo M, Martinazzo G, Prefaut C. Effects of controlled mechanical ventilation on respiratory muscle contractile properties in rabbits. Intensive Care Med 2003;29(1):103-110.

8. Levine S, Nguyen T, Taylor N, Friscia ME, Budak MT, Rothenberg $\mathrm{P}$, et al. Rapid disuse atrophy of diaphragm fibers in mechanically ventilated humans. N Engl J Med 2008;358(13):1327-1335.

9. Wait JL, Nahormek PA, Yost WT, Rochester DP. Diaphragmatic thickness-lung volume relationship in vivo. J Appl Physiol 1989; 67(4):1560-1568.

10. Boon AJ, Harper CJ, Ghahfarokhi LS, Strommen JA, Watson JC, Sorenson EJ. Two-dimensional ultrasound imaging of the diaphragm: quantitative values in normal subjects Muscle Nerve 2013;47(6): 884-889.

11. Boussuges A, Gole Y, Blanc P. Diaphragmatic motion studied by m-mode ultrasonography: methods, reproducibility, and normal values. Chest 2009;135(2):391-400.

12. Goligher EC, Laghi F, Detsky ME, Farias P, Murray A, Brace D, et al. Measuring diaphragm thickness with ultrasound in mechanically ventilated patients: feasibility, reproducibility and validity. Intensive Care Med 2015;41(4):642-649.

13. Vivier E, Mekontso Dessap A, Dimassi S, Vargas F, Lyazidi A, Thille AW, Brochard L. Diaphragm ultrasonography to estimate the work of breathing during non-invasive ventilation. Intensive Care Med 2012;38(5):796-803.

14. Gottesman E, McCool FD. ultrasound evaluation of the paralyzed diaphragm. Am J Respir Crit Care Med 1997;155(5):1570-1574.

15. Lerolle N, Guérot E, Dimassi S, Zegdi R, Faisy C, Fagon JY, Diehl JL. Ultrasonographic diagnostic criterion for severe diaphragmatic dysfunction after cardiac surgery. Chest 2009;135(2):401-407.

16. Grosu HB, Lee YI, Lee J, Eden E, Eikermann M, Rose K. Diaphragm muscle thinning in mechanically ventilated patients. Chest 2012;142(6): 1455-1460.

17. Matamis D, Soilemezi E, Tsagourias M, Akoumianaki E, Dimassi S, Boroli F, et al. Sonographic evaluation of the diaphragm in critically ill patients. Technique and clinical applications. Intensive Care Med 2013;39(5):801-810

This article is approved for Continuing Respiratory Care Education credit. For information and to obtain your CRCE

(free to AARC members) visit www.rcjournal.com

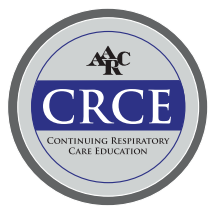

